\title{
The Nonlinear Diffusion Equation Describing Spread of Impurities of High Density
}

\author{
A.J. Janavičius, G. LüŽa And D. JuRgaitis \\ Department of Physics and Mathematics, Šiauliai University \\ Višinskio 25, Šiauliai, 5400, Lithuania
}

(Received May 6, 2004)

\begin{abstract}
The nonlinear diffusion equation is derived by taking into account the local variations in the solvent density, within a mechanism of diffusion driven by random particle collisions. Analytical solutions for the case of spherical-symmetric nonlinear diffusion equation for spread of impurities in gas and solids are obtained and discussed. In this case, the solutions of the nonlinear diffusion equation are similar to solutions of Bernoulli equation. We note that the obtained solutions can be used to describe the shapes of impurity gas of high concentration or smoke clouds.
\end{abstract}

PACS numbers: 66.30.-h

\section{Introduction}

We assume that the process of spreading of impurities is similar to other diffusion processes and, in the nonlinear case, can be described by the nonlinear diffusion equation [1-3]. In the case of three dimensions, we propose that the frequency of the jumps depends upon the particle coordinates, and the time variation of the impurities concentration $n(x, y, z, t)$ is given by [1-3]

$$
\begin{aligned}
& \frac{\partial n}{\partial t}=I_{x}+I_{y}+I_{z}, \\
& I_{x}=\nu(x+L, y, z) n(x+L, y, z)+\nu(x-L, y, z) n(x-L, y, z)-2 \nu \cdot n, \\
& I_{y}=\nu(x, y+L, z) n(x, y+L, z)+\nu(x, y-L, z) n(x, y-L, z)-2 \nu \cdot n, \\
& I_{z}=\nu(x, y, z+L) n(x, y, z+L)+\nu(x, y, z-L) n(x, y, z-L)-2 \nu \cdot n .
\end{aligned}
$$


Here, $L$ is the average free path of the particles of smoke or molecules of impurities in gases, or the length of the jump of molecules or atoms from one equilibrium position to another in fluids and solids; $\nu$ are the frequencies of these jumps or collisions. Expanding the functions $n$ and $\nu$ in the power series and including two main terms in the $\nu$ expansion and three main terms in the $n$ expansion, we obtain the following nonlinear equation:

$$
\frac{\partial n}{\partial t}=L^{2} \nu \Delta n+2 L^{2}(\operatorname{grad} \nu)(\operatorname{grad} n) .
$$

The average frequency $\nu(x, y, z, t)$ of collisions in gas is expressed as [3]

$$
\nu(x, y, z, t)=\sigma \cdot N(x, y, z, t) \cdot u \cdot \sqrt{2} .
$$

Here $\sigma$ is the collision cross-section of the diffusing particle, $N$ is the density of the solvent particles, $u \cdot \sqrt{2}$ is the relative velocity of impurity or the diffusing atoms and solvent particles. $N$ in Eq. (3) for diffusion of impurities in the crystals by vacancies is equal to the density of the vacancies.

Assuming that the coordinate $z$ is fixed, we can use the last formula in the two-dimensional case. For different processes the $N$ in Eq. (3) obtain different meanings. Usually for the diffusion processes we have $(n / N) \ll 1$, but we must take into account the fact that the concentration of solvent and impurities atoms or molecules during the diffusion process becomes $N+n$ for a non-equilibrium process for diffusion in the dense gases or $N-n$ for a diffusion process at equilibrium conditions for diffusion in the diluted solvents or in the gases of small density. For diffusion in the solids through vacancies the sum of impurities density $n$ and density of free vacancies $N$ must be equal the maximum density of vacancies $N_{\nu}$. For diffusion process in a gas it means that pressure in the solvent gas $N k T$ and the average pressure in the diffusion region $(N-n) k T+n k T$ must be equal. For this case in the region of high intensity source we can change $N$ in Eq. (3) by $n$.

Let us evaluate the second term on the right hand side of Eq. (2). In our case, only the collisions between the impurity atoms or molecules and molecules of solvent gas or vacancies in solid state materials or fluids are important. Then modelling diffusion from the source of the high intensity, we can use the approximation that for the region of diffusion front the number of a solvent particles $N_{f} \approx n / 2 \approx N / 2$. In this case we obtain

$$
\nu_{f}(x, y, z, t)=\frac{1}{2} \sigma \cdot n(x, y, z, t) \cdot u \cdot \sqrt{2} .
$$

Here we assume that a thin region adjacent to the diffusion front with a quickly decreasing impurity concentration has a half of the vacancies filled (in a fluid or solid state material), or a half of molecules replaced by impurities molecules (in a gas). Near the source all molecules of the solvent gas are replaced by molecules of impurities or all vacancies of solid state are filled with the diffusing atoms, and 
then in this region we have self-diffusion of impurities. Then we have

$$
\nu(x, y, z, t)=\sigma \cdot n(x, y, z, t) \cdot u \cdot \sqrt{2}
$$

After inserting (5) into the first term of the right hand side of Eq. (2) and changing $\operatorname{grad} \nu$ by $\operatorname{grad} \nu_{f}$ in the second term on the right hand side of Eq. (2) we obtain

$$
\frac{\partial n}{\partial t}=L^{2} \cdot \sigma \cdot u \cdot \sqrt{2}\left[n \Delta n+(\operatorname{grad} \cdot n)^{2}\right]
$$

If we define the diffusion coefficient $D$ by the following equation:

$$
D=\sqrt{2} L^{2} \sigma u n
$$

the nonlinear diffusion equation (6) becomes similar to the nonlinear equation in [2]. In both cases, the diffusion coefficient is proportional to the impurity concentration [2].

In the region of diffusion where the pressure of the impurities and of the gas is approximately the same $n$ factor in the first term of right hand side of Eq. (3) can be changed by $N$. For diffusion in solids by vacancies $N$ means the maximum density of vacancies or impurities. After inserting the obtained expression into Eq. (2), we have

$$
\frac{\partial n}{\partial t}=\sqrt{2} \cdot L^{2} \sigma N u \Delta n+\sqrt{2} L^{2} \sigma u(\operatorname{grad} \cdot n)^{2} .
$$

Earlier we proposed that on the diffusion front half of solvent particles of gas are replaced by impurities $N_{f} \approx n / 2 \approx N / 2$ but in the case of pure solvent or at the region of source $N$ is constant. In this case the diffusion coefficient can be expressed as

$$
D_{S}=\sqrt{2} \cdot L^{2} \sigma N u, \quad D_{S}=2 D .
$$

The coefficient $D_{S}$ can be compared with the diffusion coefficient $D$ for the region of the diffusion front.

Now from (8) and (9) we can obtain the following nonlinear diffusion equation for diffusion of impurities of high density:

$$
\frac{\partial n}{\partial t}=D_{S} \Delta n+\frac{D_{S}}{N}(\operatorname{grad} \cdot n)^{2}
$$

Without any doubt, this equation is also valid in one- and two-dimensional cases. In the three-dimensional spherical case the latter equation will be

$$
\frac{\partial n}{\partial t}=D_{S}\left(\frac{\mathrm{d}^{2} n}{\mathrm{~d} r^{2}}+\frac{2}{r} \cdot \frac{\mathrm{d} n}{\mathrm{~d} r}\right)+\frac{D_{S}}{N}\left(\frac{\mathrm{d} n}{\mathrm{~d} r}\right)^{2}
$$




\section{The analytical solution of the nonlinear diffusion equation}

Using the similarity variable

$$
\xi=\frac{r}{\sqrt{D_{S} t}},
$$

we transform Eq. (11) into the following form:

$$
\frac{\mathrm{d}^{2} n}{\mathrm{~d} \xi^{2}}+\left(\frac{1}{2} \xi+\frac{2}{\xi}\right) \cdot \frac{\mathrm{d} n}{\mathrm{~d} \xi}+\frac{1}{N}\left(\frac{\mathrm{d} n}{\mathrm{~d} \xi}\right)^{2}=0 .
$$

Changing,

$$
f=\frac{1}{\mathrm{~d} n / \mathrm{d} \xi},
$$

we obtain an equation that is similar to the Bernoulli equation [4]

$$
\frac{\mathrm{d} f}{\mathrm{~d} \xi}-\left(\frac{1}{2} \xi+\frac{2}{\xi}\right) \cdot f-\frac{1}{N}=0 .
$$

Below, we propose a convenient method for solving the obtained equation. First, we obtain the asymptotic solution $f_{0}$ of Eq. (15) when $\xi \rightarrow 0$

$$
f_{0}=C_{1} \xi^{2} .
$$

Then we attempt to find the general solution in the following form:

$$
f=C(\xi)_{1} \xi^{2} .
$$

Substituting the latter expression into (15), we obtain the equation

$$
\xi^{2} \frac{\mathrm{d} C(\xi)_{1}}{\mathrm{~d} \xi}-\frac{1}{2} \xi^{3} C(\xi)_{1}-\frac{1}{N}=0,
$$

which can be solved like the Bernoulli equation [4]. Then using (14) we obtain the following expression:

$$
\left[C(\xi)_{1}\right]^{-1}=\xi^{2} \frac{\mathrm{d} n}{\mathrm{~d} \xi}=N \frac{\exp \left(-\xi^{4} / 8\right)}{\int_{\infty}^{\xi} \exp \left(-\xi_{1}^{4} / 8\right) \mathrm{d} \xi_{1}+C N} .
$$

Here, we have applied the relation [5]

$$
\int \exp \left(-\xi^{4} / 8\right) \mathrm{d} \xi=\int_{\infty}^{\xi} \exp \left(-\xi_{1}^{4} / 8\right) \mathrm{d} \xi_{1}
$$

The integration constant $C$ can be defined according to the first Fick's law. The amount of impurities that was introduced at the initial point by the time $t_{0}$ is

$$
\Delta M(0, t)=-D_{S} t_{0} 4 \pi \cdot \lim _{r \rightarrow 0} r^{2} \frac{\mathrm{d} n(r, t)}{\mathrm{d} r}, \quad 0 \leq t_{0} \leq t .
$$

The amount of impurities that has passed through the surface of a spherical source with radius $R$ by the same time can be expressed as 


$$
\Delta M(R, t)=-\left.D_{S} t_{0} 4 \pi \cdot R^{2} \frac{\mathrm{d} n(r, t)}{\mathrm{d} r}\right|_{r=R}, \quad 0 \leq t_{0} \leq t .
$$

Taking into account the equality

$$
\int_{\infty}^{0} \exp \left(-\xi^{4} / 8\right) \mathrm{d} \xi=-1.524
$$

as well as (12) and (19), we obtain

$$
r^{2} \frac{\mathrm{d} n}{\mathrm{~d} r}=\sqrt{D_{S} t} \xi^{2} \frac{\mathrm{d} n}{\mathrm{~d} \xi}, \quad \lim _{\xi \rightarrow 0} \xi^{2} \frac{\mathrm{d} n}{\mathrm{~d} \xi}=\frac{N}{-1.524+C N} .
$$

Using (19), (21), (23), and (24), we obtain the integration constant $C$, which in this case can be expressed in the following form:

$$
C_{0}=-\frac{4 \pi D_{S} t_{0} \sqrt{D_{S} t}}{\Delta M_{0}}+\frac{1.524}{N}, \quad \Delta M_{0}=\Delta M(0, t) / t_{0},
$$

where $\Delta M_{0}$ is constant.

Using the first Fick's law and (19), from (25) we can obtain the quantity of molecules or particles of impurities $\Delta M_{r}=\Delta M(r, t) / t_{0}$, which spread by the time $t_{0}$ through the spherical surface $4 \pi \cdot r^{2}$ when the diffusion process occurs

$$
\begin{array}{r}
\Delta M_{r}=-C_{D} N \frac{\exp \left(-\xi^{4} / 8\right)}{\int_{\infty}^{\xi} \exp \left(-\xi_{1}^{4} / 8\right) \mathrm{d} \xi_{1}+1.524-C_{D} N / \Delta M_{0}}, \\
C_{D}=4 \pi D_{S} \sqrt{D_{S} t}
\end{array}
$$

In order to have positive flux through all spherical surfaces, the following condition must be satisfied in the latter formula:

$$
\int_{\infty}^{\xi} \exp \left(-\xi_{1}^{4} / 8\right) \mathrm{d} \xi_{1}+1.524-C_{D} \frac{N}{\Delta M_{0}}<0 .
$$

For all finite values of $\xi$, the integral in the latter expression is negative, and when $\xi \rightarrow \infty$ it equals zero. Then this inequality will be satisfied for all values of $\xi$ and $r$ when

$$
C_{D} \frac{N}{\Delta M_{0}}>1.524
$$

Only by introducing the following limited quantity of impurity particles or molecules

$$
\Delta M_{0}<C_{D} \frac{N}{1.524}
$$

we obtain the propagation of impurities solely by diffusion. 
After integrating (19), we obtain the distribution of the radial density $n(r, t)$ of particles introduced by diffusion from the point source

$$
n(r, t)=N \int_{\infty}^{\xi} \frac{\xi_{1}^{-2} \exp \left(-\xi_{1}^{4} / 8\right)}{\int_{\infty}^{\xi_{1}}\left(-\xi_{2}^{4} / 8\right) \mathrm{d} \xi_{2}+1.524-4 \pi\left(D_{S} t\right)^{3 / 2} N / \Delta M(0, t, t)} \mathrm{d} \xi_{1}
$$

The above formula shows that the concentration of smoke in the medium decreases as $\exp \left(-r^{4} / 8 D_{S}^{2} t^{2}\right)$ and depends on the diffusion coefficient $D_{S}$ and on the amount of impurities $\Delta M(0, t)$ that was introduced during the time $t$ from the pollution source.

Now, using (19), (22), (23), (24), and (25), and taking into account that the amount of impurities $\Delta M\left(R, t_{0}, t\right)$ that has passed through the surface $4 \pi R^{2}$ of source by the time $t_{0}$ is known (see (22)), we obtain the integration constant in (19)

$$
\begin{gathered}
C_{R}=-4 \pi D_{S} t_{0} \sqrt{D_{S} t} \frac{1}{\Delta M(R, t)} \exp \left(-\xi_{R}^{4} / 8\right)-\frac{1}{N} \int_{\infty}^{\xi_{R}} \exp \left(-\xi_{1}^{4} / 8\right) \mathrm{d} \xi_{1} \\
\xi_{R}=\frac{R}{\sqrt{D_{S} t}} .
\end{gathered}
$$

Here, we assumed that the amount of impurities introduced per unit time $\Delta M(R, t) / t_{0}$ is constant.

Now, from (22) and (19) we obtain

$$
\Delta M(r, t)=-4 \pi D_{S} t_{0} \sqrt{D_{S} t} N \frac{\exp \left(-\xi^{4} / 8\right)}{\int_{\infty}^{\xi} \exp \left(-\xi_{1}^{4} / 8\right) \mathrm{d} \xi_{1}+C_{R} N}, \quad r \geq R
$$

By integrating (19), we can now determine the distribution of the radial density $n(r, t)_{R}$ of impurities introduced by diffusion through the spherical surface $4 \pi R^{2}$ of source

$$
n(r, t)_{R}=N \int_{\infty}^{\xi}\left(\frac{\xi_{1}^{-2} \exp \left(-\xi_{1}^{4} / 8\right)}{\int_{\infty}^{\xi_{1}}\left(-\xi_{2}^{4} / 8\right) \mathrm{d} \xi_{2}+C_{R} N}\right) \mathrm{d} \xi_{1}, \quad \xi \leq \xi_{R}
$$

The flux of impurities (32) is positive only when

$$
\Delta M(0, t) \int \exp \left(-\xi^{4} / 8\right) \mathrm{d} \xi-4 \pi\left(D_{S} t\right)^{3 / 2} N<0 .
$$

Hence, after replacement in $(32) \xi \rightarrow 0$, the following conditions for physical spreading of impurities by the diffusion process are obtained

$$
\Delta M(0, t)<2.625 \pi\left(D_{S} t\right)^{3 / 2} N .
$$


Using formulae for differentiating of integrals [5] and substituting (34) into (30), we obtain a useful expression for radial density of diffusing impurities

$$
n(r, t)=N \int_{\infty}^{\xi} \frac{\xi_{2}^{-2} \exp \left(-\xi_{2}^{4} / 8\right) \mathrm{d} \xi_{2}}{\int_{\infty}^{\xi_{2}}\left(-\xi_{1}^{-4} / 8\right) \mathrm{d} \xi_{1}-4 \pi\left(D_{S} t\right)^{3 / 2} N / M(0, t)} .
$$

The concentration field surrounding the spherical source of radius $R$ can also be determined by spherical diffusion solution of linear diffusion equation [6]

$$
C_{\alpha}(r, t)=C_{\alpha / \beta} \frac{R}{r} \operatorname{erfc}\left(\frac{r-R}{2 \sqrt{D_{S} t}}\right), \quad r \geq R
$$

with the boundary condition $\lim _{r \rightarrow R} C_{\alpha}(r, t)=C_{\alpha / \beta}$.

\section{Results and conclusions}

The obtained solutions (31), (35) can be appropriate for describing the clouds of smoke in the air or density of impurities in gases and solids. The obtained solutions of the nonlinear diffusion equation (13) define the impurity flux and their radial density in the spherical case. By substituting (31) into (32), we can express the amount $\Delta M(r, t)$ of molecules or particles introduced by the diffusion process through the surface $4 \pi r^{2}$ by the time $t_{0}$ in the following way:

$$
\begin{gathered}
\Delta M(r, t)=\frac{-4 \pi D_{S} t_{0} \sqrt{D_{S} t} N \cdot \exp \left(-\xi^{4} / 8\right)}{\int_{\xi_{R}}^{\xi} \exp \left(-\xi_{1}^{4} / 8\right) \mathrm{d} \xi_{1}-4 \pi D_{S} t_{0} \sqrt{D_{S} t} \frac{N}{\Delta M(R, t)} \exp \left(-\xi_{R}^{4} / 8\right)}, \\
r \geq R .
\end{gathered}
$$

The presented solution satisfies the boundary condition for the amount of impurities at the spherical source of radius $R$ when $r \rightarrow R$ or $\xi \rightarrow \xi_{R}$

$$
\lim _{r \rightarrow R} \Delta M(r, t)=\Delta M(R, t) \text {. }
$$

Using (21) and (37), we can also define the amount of molecules or particles $\Delta M L(r, t)$ introduced by the diffusion process through the surface $4 \pi r^{2}$ by the time $t_{0}$ for the solution (37) of the linear diffusion equation

$$
\Delta M L(r, t)=4 \pi r^{2} t_{0} \cdot D \cdot C_{\alpha / \beta}\left[\frac{R}{r \sqrt{\pi D t}}+\frac{R}{r^{2}} \operatorname{erfc}\left(\frac{r-R}{\sqrt{4 D t}}\right)\right] .
$$

By requiring that fluxes at the source of impurities for the cases (38) and (40) are the same

$$
\lim _{r \rightarrow R} \Delta M L(r, t)=\Delta M(R, t)
$$

we obtain

$$
C_{\alpha / \beta}=\frac{\Delta M(R, t)}{4 \pi R^{2} t_{0} D\left(\frac{1}{\sqrt{\pi D t}}+\frac{1}{R}\right)} .
$$




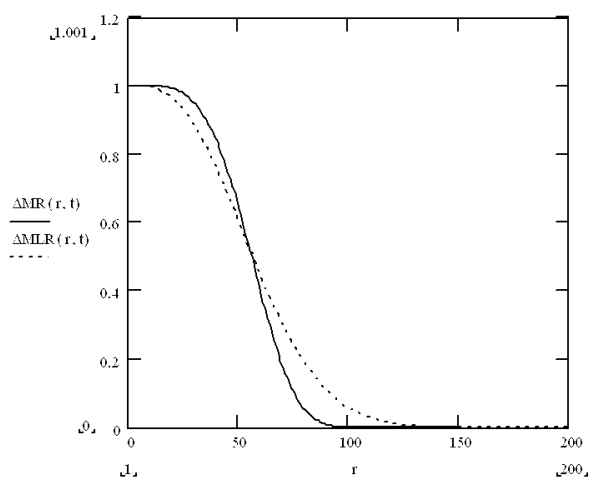

Fig. 1. Dependence of nonlinear $\Delta M R(r, t)$ and linear $\Delta M L R(r, t)$ relative diffusion flows by time $t_{0}=1 \mathrm{~s}$ on the distance $r \mathrm{~cm}$ when diffusion time $t$ is $3600 \mathrm{~s}$. The radius of the source is $R=1 \mathrm{~cm}$. The amount of impurity particles introduced per unit time $\Delta M(R, t) / t_{0}=2.68 \times 10^{19} / \mathrm{s}$ and the density of the air molecules $N=2.68 \times 10^{19} \mathrm{~cm}^{-3}$ are constant. The diffusion coefficient for linear diffusion of $\mathrm{CO}$ in the air [8] is $D=$ $0.182 \mathrm{~cm}^{2} / \mathrm{s}$ and for nonlinear diffusion it is $D_{S}=0.364 \mathrm{~cm}^{2} / \mathrm{s}$.

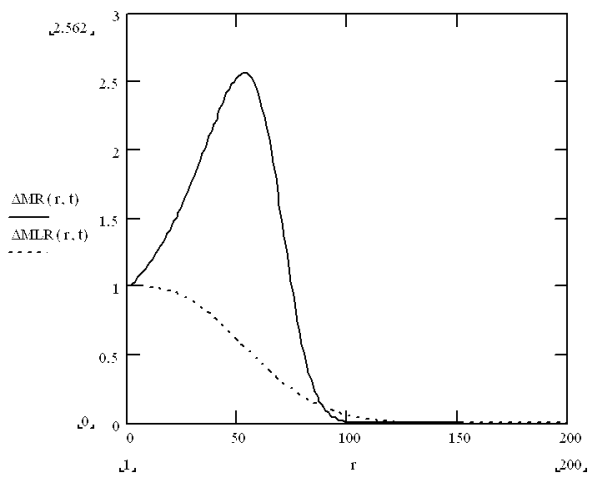

Fig. 2. Dependence of nonlinear $\Delta M R(r, t)$ and linear $\Delta M L R(r, t)$ relative diffusion flows by the time $t_{0}=1 \mathrm{~s}$ on the distance $r \mathrm{~cm}$ when diffusion time $t$ is $3600 \mathrm{~s}$. The radius of the source is $R=1 \mathrm{~cm}$. The amount of impurity particles introduced per unit time $\Delta M(R, t) / t_{0}=2.68 \times 10^{21} / \mathrm{s}$ and density of the air molecules $N=2.68 \times 10^{19} \mathrm{~cm}^{-3}$ are constant. The diffusion coefficients for linear diffusion of $\mathrm{CO}$ in the air [8] is $D=$ $0.182 \mathrm{~cm}^{2} / \mathrm{s}$ and for nonlinear diffusion it is $D_{S}=0.364 \mathrm{~cm} / \mathrm{s}$.

The nonlinear $\Delta M R(r, t)=\Delta M(r, t) / \Delta M(R, t)$ and linear $\Delta M L R(r, t)=$ $\Delta M L(r, t) / \Delta M(R, t)$ relative flows, which give the relative amounts of $\mathrm{CO}$ in the air, calculated using (38), (40), and (42), are presented in Fig. 1.

The results presented in Fig. 1 show that nonlinear $\Delta M R(r, t)$ and linear $\Delta M L R(r, t)$ flows significantly differ only at long distances. In the nonlinear diffusion model (6) obtained using method proposed in [3] where the average length $L$ 
of jump of molecules or atoms from one equilibrium position to another was introduced. The similar nonlinear diffusion equation was obtained in [2] requiring that the diffusion processes can occur only with a finite velocity, which is essentially the same assumption.

We must remark that we solved Eq. (10) that is of some approach to (6). Equation (6) was successfully used in paper [7] for definition of profiles of high concentration diffusion profiles of boron and phosphorus in the crystal silicon.

Interesting results, which were obtained in the case of a large amount of $\mathrm{CO}$ molecules introduced per unit time $\left(\Delta M(R, t) / t_{0}=2.68 \times 10^{21} / \mathrm{s}\right)$, are presented in Fig. 2. In the nonlinear case at $50 \mathrm{~cm}$ we obtained $\Delta M R(r, t)=\Delta M(r, t) / \Delta M(R, t)$ with a local maximum, i.e., a ring of impurities.

It is interesting to note that the above-mentioned rings of impurities cannot be obtained in the linear case (40). These rings of increased density of impurities can be obtained when source can introduce a large amount of impurities by short time.

\section{References}

[1] A.J. Janavičius, Random Processes and Transfer Phenomena, Šiauliai University, Šiauliai 2002, p. 85 (in Lithuanian).

[2] A.J. Janavičius, Phys. Lett. A 224, 159 (1997).

[3] B.-F. Apostol, Phys. Lett. A 235, 363 (1997).

[4] P.F. Filčakov, Handbook of High Mathematics, Naukova Dumka, Kiev 1973, p. 743 (in Russian).

[5] G. Korn, T. Korn, Mathematical Handbook for Scientists and Engineers McGRAW-HILL Book Company, New York 1973, p. 684

[6] M.E. Gliksman, Diffusion in Solids, John Wiley \& Sons, New York 2000, p. 472.

[7] W.-S. Wang, Y.-H. Lo, IEEE Trans. Electron Devices ED-30, 1828 (1983).

[8] Laboratory Guide of Physics, Ed. L.L. Goldin, Nauka, Moscow 1983, p. 704 (in Russian). 